\title{
A Combinatorial Proof of a Schmidt Type Theorem of Andrews and Paule
}

\author{
Kathy Q. Ji \\ Center for Applied Mathematics \\ Tianjin University \\ Tianjin 300072, P.R. China \\ kathyji@tju.edu.cn
}

Submitted: Nov 1, 2021; Accepted: Jan 20, 2022; Published: Feb 11, 2022

(C) The author. Released under the CC BY-ND license (International 4.0).

\begin{abstract}
This note is devoted to a combinatorial proof of a Schmidt type theorem due to Andrews and Paule. A four-variable refinement of Andrews and Paule's theorem is also obtained based on this combinatorial construction.
\end{abstract}

Mathematics Subject Classifications: 05A17, 11P81

The main objective of this note is to give a combinatorial proof of the following partition theorem due to Andrews and Paule [2]. Sylvester's bijection [3, 4, 5] for Euler's partition theorem and Wright's bijection $[1,6,7]$ for Jacobi's triple product identity play important roles in the combinatorial construction.

Theorem 1 (Andrews-Paule). Assume that $n \geqslant 1$. Let $s(n)$ denote the number of partitions $a_{1}+a_{2}+a_{3}+\cdots$ satisfying $a_{1} \geqslant a_{2} \geqslant a_{3} \geqslant \cdots$ and $n=a_{1}+a_{3}+a_{5}+\cdots$. Let $t(n)$ denote the number of two-color partitions of $n$. Then

$$
s(n)=t(n)
$$

For example, let $n=3$. There are ten partitions counted by $s(3)$, which are

$$
\begin{aligned}
& 3,3+3,3+2,3+1,2+2+1,2+2+1+1,2+1+1,2+1+1+1, \\
& 1+1+1+1+1,1+1+1+1+1+1 .
\end{aligned}
$$

and there are also ten red and green partitions counted by $t(3)$, which are

$$
\begin{aligned}
& 3_{r}, 3_{g}, 2_{r}+1_{r}, 2_{g}+1_{r}, 2_{r}+1_{g}, 2_{g}+1_{g}, 1_{r}+1_{r}+1_{r}, 1_{r}+1_{r}+1_{g}, \\
& 1_{r}+1_{g}+1_{g}, 1_{g}+1_{g}+1_{g} .
\end{aligned}
$$


Proof of Theorem 1. Let $\mathcal{S}(n)$ denote the set of partitions counted by $s(n)$ and let $\mathcal{T}(n)$ denote the set of two-color partitions counted by $t(n)$. We aim to construct a bijection $\phi$ between $\mathcal{T}(n)$ and $\mathcal{S}(n)$.

Let $\lambda$ be a two-color partition in $\mathcal{T}(n)$ with $r$ red parts and $l$ green parts. Assume that $m=\max \{r, l\}$. We aim to define $\phi(\lambda)=\gamma=\left(\gamma_{1}, \gamma_{2}, \ldots, \gamma_{2 m-1}, \gamma_{2 m}\right)$ such that $\gamma_{1} \geqslant \gamma_{2} \geqslant \ldots \geqslant \gamma_{2 m-1} \geqslant \gamma_{2 m} \geqslant 0$ and $\gamma_{1}+\gamma_{3}+\cdots+\gamma_{2 m-1}=n$.

Let $\alpha$ be a partition consisting of all red parts in $\lambda$ and $\beta$ be a partition consisting of all green parts in $\lambda$. First, add 0 at the end of $\alpha$ or $\beta$ so that they are of the same length depending on which is of smaller length. Assume that $r \leqslant l$, so $m=l$. Then $\alpha=(\alpha_{1}, \alpha_{2}, \ldots, \alpha_{r}, \underbrace{0, \ldots, 0}_{l-r})$ and $\beta=\left(\beta_{1}, \beta_{2}, \ldots, \beta_{l}\right)$.

We next define a pair $(\bar{\alpha}, \bar{\beta})$ of partitions with distinct parts corresponding to $(\alpha, \beta)$, where $\bar{\alpha}=\left(\alpha_{1}+l-1, \alpha_{2}+l-2, \ldots, \alpha_{r}+l-r, l-r-1, \ldots, 1,0\right)$, and $\bar{\beta}=\left(\beta_{1}+l-\right.$ $\left.1, \beta_{2}+l-2, \ldots, \beta_{l}\right)$. Obviously, $|\bar{\alpha}|+|\bar{\beta}|=|\alpha|+|\beta|+l(l-1)$.

We now apply Wright's bijection to represent $(\bar{\alpha}, \bar{\beta})$ as a Young diagram of an ordinary partition $Y(\bar{\alpha}, \bar{\beta})$ : put $l$ squares on the diagonal, and then for $j=1,2, \ldots, l$, put $\bar{\alpha}_{j}$ squares in the $j$-th row to the right of the diagonal and $\bar{\beta}_{j}$ squares in the $j$-th column below the diagonal. For example, Figure 1. gives the Young diagram of $(\bar{\alpha}, \bar{\beta})$, where $\bar{\alpha}=(3,2,0)$ and $\bar{\beta}=(5,3,1)$.

The Young diagram

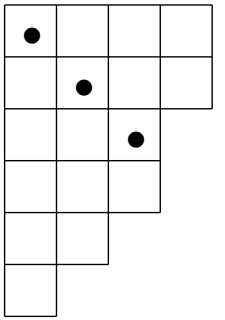

2-modular diagram

\begin{tabular}{|l|l|l|l|}
\hline 2 & 2 & 2 & 1 \\
\hline 2 & 2 & 2 & 1 \\
\hline 2 & 2 & 1 & \multicolumn{1}{|c}{} \\
\cline { 1 - 2 } 2 & 2 & 1 & \multicolumn{1}{|c}{} \\
\cline { 1 - 2 } 2 & 1 & \multicolumn{2}{|c}{} \\
\cline { 1 - 2 } 1 & \multicolumn{2}{|c}{} \\
\cline { 1 - 2 } & &
\end{tabular}

Figure 1: The Young diagram of $Y(\bar{\alpha}, \bar{\beta})$ and 2-modular diagram.

For each row in the Young diagram of $(\bar{\alpha}, \bar{\beta})$, write 2 in each box and a 1 at the end of the row to obtain the 2-modular diagram. Decompose the 2-modular diagram into hooks $H_{1}, H_{2}, \ldots$ with the diagonal boxes as corners. Let $\mu_{1}$ be the number of squares in $H_{1}$, let $\mu_{2}$ be the number of 2's in $H_{1}$, let $\mu_{3}$ be the number of squares in $H_{2}$, let $\mu_{4}$ be the number of 2's in $H_{2}$, and so on. Set $\mu=\left(\mu_{1}, \mu_{2}, \ldots, \mu_{2 l-1}, \mu_{2 l}\right)$, see Figure 2 . Then $\mu$ is clearly a partition with distinct parts. Furthermore, $\mu_{1}+\mu_{3}+\cdots+\mu_{2 l-1}=$ $|\alpha|+|\beta|+l^{2}$. Hence we may define $\gamma=\left(\mu_{1}-(2 l-1), \mu_{2}-2 l-2, \ldots, \mu_{2 l-1}-1, \mu_{2 l}\right)$. Clearly, $\gamma_{1}+\gamma_{3}+\cdots+\gamma_{2 l-1}=|\alpha|+|\beta|$, and so $\gamma \in \mathcal{S}(n)$. Furthermore, this process is reversible since Sylvester's bijection and Wright's bijection are reversible. Thus, we complete the proof of Theorem 1.

Applying the above bijection, we get the following correspondence between the set 
2-Modular Diagram

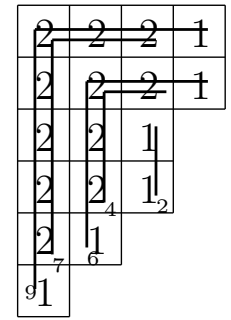

$\lambda$

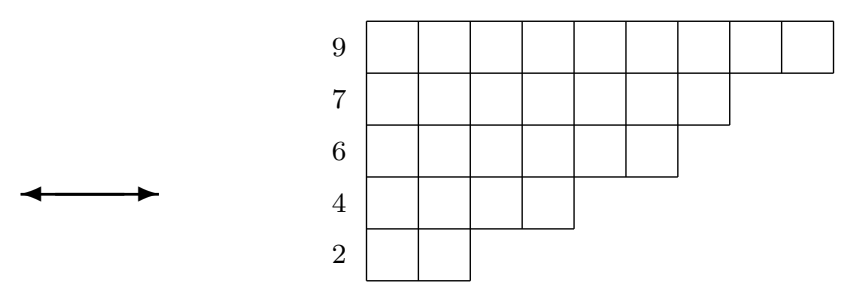

$\mu$

Figure 2: Sylvester's bijection.

$\mathcal{T}(3)$ and the set $\mathcal{S}(3)$.

$$
\begin{array}{ccc}
3_{r} \leftrightarrows 3+3 & 3_{g} \leftrightarrows 3 & 2_{r}+1_{r} \leftrightarrows 2+2+1+1 \\
2_{g}+1_{r} \leftrightarrows 3+1 & 2_{r}+1_{g} \leftrightarrows 3+2 & 2_{g}+1_{g} \leftrightarrows 2+1+1 \\
1_{r}+1_{r}+1_{r} \leftrightarrows 1+1+1+1+1+1 & 1_{r}+1_{r}+1_{g} \leftrightarrows 2+1+1+1 \\
1_{r}+1_{g}+1_{g} \leftrightarrows 2+2+1 & 1_{g}+1_{g}+1_{g} \leftrightarrows 1+1+1+1+1
\end{array}
$$

The following result immediately follows from the combinatorial construction of Theorem 1.

Theorem 2. Assume that $n \geqslant 1, r, l, p, q \geqslant 1$. Let $s_{r, l, p, q}(n)$ denote the number of partitions $a_{1}+a_{2}+a_{3}+\cdots+a_{2 \max \{r, l\}}$ satisfying $p+q \geqslant a_{1} \geqslant a_{2} \geqslant \cdots \geqslant a_{2 \max \{r, l\}} \geqslant 0$ and $n=a_{1}+a_{3}+a_{5}+\cdots+a_{2 \max \{r, l\}-1}$. Let $t_{r, l, p, q}(n)$ denote the number of two-color partitions of $n$ such that there are $r$ red parts and $l$ blue parts with the largest red part being not larger than $p$ and the largest blue part being not larger than $q$. Then

$$
s_{r, l, p, q}(n)=t_{r, l, p, q}(n)
$$

\section{Acknowledgements}

This work was supported by the National Science Foundation of China. We wish to thank the referees for valuable suggestions.

\section{References}

[1] G.E. Andrews, Generalized Frobenius partitions, Mem. Amer. Math. Soc. 49 (1984) No. 301, iv+, 44 pp.

[2] G. E. Andrews and P. Paule, MacMahon's partition analysis XIII: Schmidt type partitions and modular forms, J. Number Theory, (2021), https://doi.org/10.1016/j.jnt.2021.09.008. 
[3] C. Bessenrodt, A bijection for Lebesgue's partition identity in the spirit of Sylvester, Discrete Math. 132 (1994) 1-10.

[4] D. Bressoud, Proofs and Confirmations: The story of the alternating sign matrix conjecture, Cambridge University Press, 1999.

[5] P. A. MacMahon, Combinatory Analysis, Vol. II, Cambridge University Press, Cambridge, 1915-1916, Reprinted: Chelsea, New York, 1960.

[6] A.J. Yee, Combinatorial proofs of generating function identities for $F$-partitions, J. Combin. Theory Ser. A 102 (2003) 217-228.

[7] E.M. Wright, An enumerative proof of an identity of Jacobi, J. London Math. Soc. 40 (1965) 55-57. 